\title{
A Spin-Labelled Liquid Crystal. A New Spin Probe for the Study of the Orientational Order and Molecular Dynamics of Liquid Crystals
}

\author{
G. R. Luckhurst, S. W. Smith and F. Sundholm*, \\ Department of Chemistry, The University, Southampton S095NH, U.K.
}

\begin{abstract}
Luckhurst, G. R., Smith, S. W. and Sundholm, F., 1987. A Spin-Labelled Liquid Crystal. A New Spin Probe for the Study of the Orientational Order and Molecular Dynamics of Liquid Crystals. - Acta Chem. Scand., Ser. A 41: 218-229.
\end{abstract}

\begin{abstract}
A new spin probe has been prepared by spin-labelling the liquid crystal 4- $n$-butoxy-4'-acetylazobenzene (1) with an oxazolidine- $N$-oxyl group in the $\alpha$-position of the acetyl side chain. The potential of this spin probe, $4-n$-butoxy-4'-ethyl$\left(4^{\prime \prime}, 4^{\prime \prime}\right.$-dimethyl-3"- $N$-oxyloxazolidine)azobenzene (2), has been demonstrated by employing it to study the orientational order and molecular dynamics in the parent liquid crystal, 1. The line positions in the ESR spectrum reflect the second rank order parameter for the long axis of the spin probe. However, the determination of this order parameter requires the angle between the molecular long axis and the axis orthogonal to the plane of the oxazolidine ring; structural studies suggest that the angle is approximately $30^{\circ}$. The linewidths also depend on this angle, and a comparison of the observed and predicted linewidths proves to be consistent with an angle of about $30^{\circ}$. The rotational correlation time for the molecular long axis of the spin probe dissolved in the smectic A phase of 1 is available from our analysis of the linewidths and is found to be approximately $10^{-9} \mathrm{~s} \mathrm{rad}^{-1}$.
\end{abstract}

Nitroxide free radicals are widely used to probe the orientational order and molecular dynamics of liquid crystals. ${ }^{1}$ The spectral analysis is often straightforward because the mesophase is sufficiently mobile for the motion of the spin probe to be in the fast motion regime. In consequence, the line positions in the spectrum yield the second rank order parameter, $P_{2}$, and the linewidths can be used to obtain the fourth rank order parameter, $\overline{P_{4}}$, as well as the rotational correlation times characterising the dynamics. ${ }^{2}$ Since ESR spectroscopy is a particularly sensitive technique, the concentration of the spin probe can be extremely small $\left(<10^{-5} \mathrm{M}\right)$ and so the molecular organisation within the liquid crystal should not be seriously perturbed by the introduction of the probe. None the less, it is important to recognise that the ESR measurements yield the order par-

\footnotetext{
${ }^{*}$ Present and permanent address: Department of Chemistry, University of Helsinki, Et. Hesperiank. 4, SF-00100 Helsinki, Finland.

${ }^{\S}$ To whom correspondence should be addressed.
}

ameters and dynamics of the spin probe and not of the mesogenic molecules. Of course, such properties are determined by solute-solvent interactions together with the solvent order and it is possible to relate these to the solute order with the aid of theory. ${ }^{3}$

If, however, the behaviour of the spin probe is to approach that of the mesogenic host then they should have similar molecular structures. In principle, this should be relatively easy to achieve because the chemical stability of the nitroxide moiety permits the synthesis of a wide range of structures. In practise, there have been few studies of thermotropic liquid crystals using nitroxide spin probes with structures strictly comparable to that of the solvent. ${ }^{4}$ We have therefore spin-labelled a liquid crystal to create a spin probe which should prove valuable for the investigation of many analogous thermotropic mesogens. The spin probe was prepared by introducing an oxazolidine- $N$-oxyl group in the $\alpha$-position of the acetyl function of the mesogen 4- $n$-butoxy-4'acetylazobenzene (1); the molecular structures of 


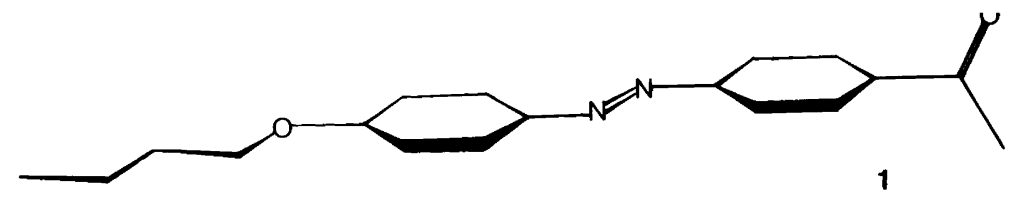

1

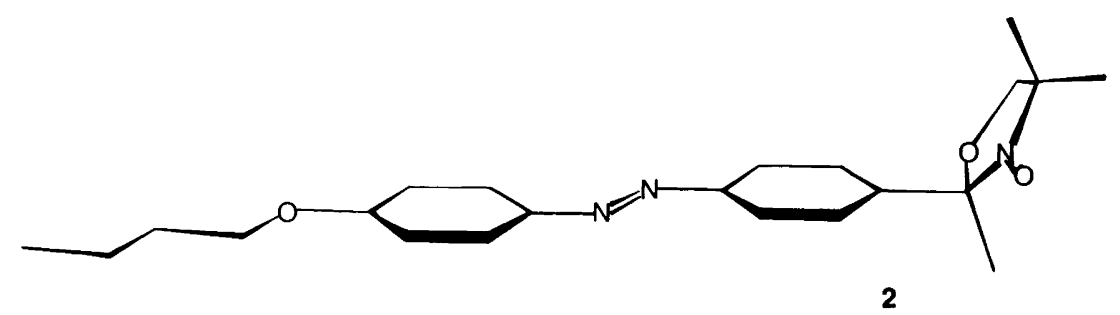

Fig. 1. The structural formulas of the mesogen $4-n$-butoxy-4'-acetylazobenzene (1) and the spin probe 4-n-butoxy-4'-ethyl-(4",4"-dimethyl-3"-N-oxyloxazolidine)azobenzene (2).

the liquid crystal and the spin probe, 2 , are shown in Fig. 1. The potential of the spin probe was then explored by using it to study the nematic and smectic A phases of the parent mesogen.

In the following sections we describe the synthesis of the spin probe, 2 , together with the ESR measurements on the solution of the probe in the mesogen, 1. The second rank orientational order parameter of the probe in the nematic and smectic A phases of $\mathbf{1}$ is determined from the nitrogen hyperfine spacing; the assumptions needed in this analysis are explained. As we shall see, we require a knowledge of the molecular geometry of the probe. The same information is also needed in the interpretation of the angular variation of the linewidths in the smectic A phase to yield the fourth rank order parameter and the rotational correlation times. However, we shall use our results to show that the line positions and their widths are consistent with the same molecular geometry. Finally, the linewidths allow us to estimate the rotational correlation time for the molecular long axis of the probe dissolved in the smectic A phase of the mesogen.

\section{Experimental}

Synthesis. The liquid crystal, $\mathbf{1}$, was prepared by diazo coupling between $n$-butoxybenzene and 4-aminoacetophenone. The transition temperatures of the purified mesogen were determined by differential thermal analysis (du Pont) as

$$
\mathrm{C} \stackrel{109.1^{\circ} \mathrm{C}}{\longrightarrow} \mathrm{S}_{\mathrm{A}} \stackrel{112.5^{\circ} \mathrm{C}}{\longrightarrow} \mathrm{N} \stackrel{122.2^{\circ} \mathrm{C}}{\longrightarrow} \mathrm{I}
$$

they are in good agreement with literature values. ${ }^{5}$ The spin probe, 2 , was synthesised by heating 2-amino-2-methylpropanol $(20 \%$ excess $)$ with the mesogen, 1 , for $18 \mathrm{~h}$ at $105^{\circ} \mathrm{C}$, without a solvent or a catalyst. The reaction mixture was poured into water and extracted thoroughly with toluene. The toluene solution was washed with dilute sulfuric acid and dried over sodium sulfate. The resultant amine was then oxidized to the nitroxide by agitation with a $20 \%$ excess of $m$-chloroperbenzoic acid overnight at room temperature. The toluene solution was washed with aqueous sodium hydroxide, dried and the toluene removed by evaporation in vacuo. The product was recrystallised from chloroform to give a material which melted in the range $86-90^{\circ} \mathrm{C}$. The spin probe is not mesomorphic since, presumably, the bulky oxazolidine- $N$-oxyl group decreases the length-to-breadth ratio and so depresses the nematic-isotropic transition below the melting point. However, higher members of the homologous series with their longer terminal chains may well be liquid-crystalline. 
Characterisation. The liquid crystal 1 was characterised by proton NMR spectroscopy and by differential scanning calorimetry (Perkin-Elmer2B).

ESR measurements. The liquid crystal, doped with trace quantities of the spin probe, was sealed in a $4 \mathrm{~mm}$ (o.d.) Pyrex tube after degassing the sample to prevent line-broadening by dissolved oxygen. The sample tube was fitted into-a goniometer attached to the microwave cavity of a Varian E3 ESR spectrometer. The temperature of the sample was accurately controlled by pumping oil from a Lauda thermostat through a quartz jacket. ${ }^{6}$ The temperature was monitored with a copper/constantan thermocouple located adjacent to the sample tube and whose output was measured with a Hewlett-Packard digital-multimeter; the temperature was found to be stable to $\pm 0.04^{\circ} \mathrm{C}$ during the time taken to record a series of spectra. The klystron frequency was measured with a Hewlett-Packard frequency counter and observed to vary slightly from $9.1274 \mathrm{GHz}$ in the isotropic phase to $9.1267 \mathrm{GHz}$ in the liquid-crystalline phase; in the linewidth analysis it was taken to be $9.127 \mathrm{GHz}$. The sample was first heated to give the isotropic phase and ESR spectra were recorded as the mesogen was cooled, giving first the nematic and then the smectic A phase. The nematic phase is aligned by the magnetic field with the director parallel to it. This alignment is preserved in the smectic A phase, provided the temperature is lowered slowly through the smectic A-nematic transition and the magnetic field is held at its maximum value of $0.6 \mathrm{~T}$. The smectic layers are then formed orthogonal to the field, so that rotation of the sample about an axis perpendicular to the field also rotates the director with respect to the field. This technique has been described in detail by Luckhurst and Setaka. ${ }^{7}$ The transition from the smectic A to the crystal can be supercooled and so it proved possible to make measurements in the smectic A phase to about $10^{\circ} \mathrm{C}$ below the melting point. In addition to these measurements with the director parallel to the field, the angular variation of the ESR spectrum of the spin probe in the smectic A phase was studied at $104.6^{\circ} \mathrm{C}$.

\section{Results and discussion}

Orientational order. The ESR spectrum of the spin probe consists of three antisymmetric nitrogen hyperfine lines in the isotropic, nematic and smectic A phases. The symmetry and width of the hyperfine lines demonstrate that the rate of molecular reorientation corresponds to the fast motion limit with a correlation time smaller than $10^{-8} \mathrm{~s} \mathrm{rad}^{-1}$. The form of the spectrum in the smectic A phase shows that despite its high bulk viscosity, the probe molecules and presumably those of the mesogenic host are reorienting rapidly within the smectic layers. For the fast motion regime, the change in the nitrogen hyperfine spacing on passing from the isotropic to a uniaxial liquid-crystalline phase with the director parallel to the magnetic field is given by ${ }^{1,8}$

$$
\bar{A}_{\|}-a=(2 / 3) \sum_{\alpha, \beta} S_{\alpha \beta} A^{\prime}{ }_{\alpha \beta} .
$$

Here, $\tilde{A}_{\|}$is the component of the partially averaged nitrogen hyperfine tensor parallel to the director, $a$ is the scalar coupling constant, $A^{\prime}$ is the anisotropic hyperfine tensor and $S$ is the Saupe ordering matrix which describes the extent of the orientational order for the probe. ${ }^{9}$ This result is valid provided the spin probe is rigid, but needs to be modified when this is not so. ${ }^{10}$ The spin probe, 2, (cf. Fig. 1) is clearly not rigid because the phenyl rings can undergo internal rotation and because of the flexibility of the $n$-butoxy chain. However, the anisotropic nitrogen hyperfine tensor, $A^{\prime}$, is not expected to change with the molecular conformation, and in this case eqn. (1) remains valid but now the ordering matrix, $S$, is an average over those for all conformations. ${ }^{10}$ The lack of high symmetry for even the conformationally averaged structure of the probe means that we are unable to locate the principal axes of the averaged ordering matrix without additional information. Fortunately, this problem is simplified because the nitrogen hyperfine tensor is, to a good approximation, cylindrically symmetric about an axis orthogonal to the plane of the oxazolidine ring. In this frame, eqn. (1) reduces to

$$
\tilde{A_{\|}}-a=A_{z^{\prime} z^{\prime}}^{\prime} S_{z^{\prime} z^{\prime}}
$$


where $z^{\prime}$ is the symmetry axis for the nitrogen hyperfine tensor. The shift in the nitrogen coupling constant yields, therefore, the order parameter for the $z^{\prime}$ axis of the spin probe. To relate this quantity to the order parameter for the molecular long axis requires some assumptions. Although the conformationally averaged structure does not possess a rotation axis with three-fold or higher symmetry, it is reasonable to assume that the deviation of the spin probe, 2 , from cylindrical symmetry is negligibly small. In other words, the averaged ordering matrix is taken to be cylindrically symmetric. With this assumption, the component of the ordering tensor, $S_{z^{\prime} z^{\prime}}$, is related to the second rank order parameter for the effective molecular symmetry axis, $\overline{P_{2}}$, by $^{8}$

$S_{z^{\prime} z^{\prime}}=\widetilde{P_{2}} P_{2}(\cos \beta)$

Here, $P_{2}(\cos \beta)$ is the second Legendre polynomial and $\beta$ is the angle between $z^{\prime}$ and the molecular long axis.

To obtain the order parameter, $P_{2}$, from the observed nitrogen hyperfine spacing, $\tilde{A}_{\|}$, in the nematic and smectic $A$ phases of 1 , the scalar coupling constant $a$ was set equal to that $(1.431 \mathrm{mT})$ in the isotropic phase. The anisotropic component $A_{z^{\prime} z^{\prime}}^{\prime}$ was determined by recording the powder spectrum of the spin probe 2 dissolved in an $o$-terphenyl glass and was found to be $1.667 \mathrm{mT}$. The angle $\beta$ was estimated to be approximately $30^{\circ}$ with the aid of molecular models, as well as from the X-ray structure of 1,4bis[spiro-(2'-N-oxyl-3', $3^{\prime}$-dimethyloxazolidine)] cyclohexane. ${ }^{11}$ The temperature dependence of the order parameter, $\overline{P_{2}}$, obtained with these assumptions is shown in Fig. 2, using a shifted temperature scale $\left(T_{\mathrm{NI}}-T\right)$. The variation of $\overline{P_{2}}$ with temperature is in accord with studies of similar mesogens using other spin probes, ${ }^{7,12,13}$ and, indeed, of pure mesogens. ${ }^{14}$ The order parameter of the probe at the nematic-isotropic transition is about 0.24 , which is slightly lower than that observed for nematogens; this slight reduction is compatible with the increase in the molecular breadth caused by the bulky oxazolidine group; however, the value of the order parameter is, in addition, rather sensitive to the choice of $\beta$. As the temperature is lowered $\overline{P_{2}}$ increases rapidly within the nematic phase, and at the smectic A-
A SPIN-LABELLED LIQUID CRYSTAL

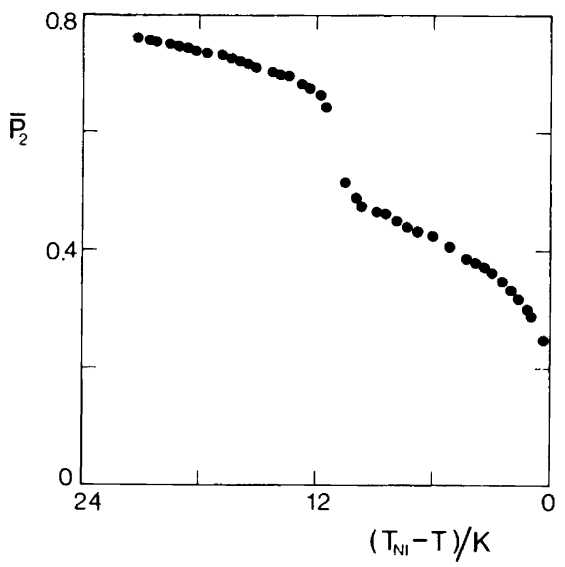

Fig. 2. The dependence of the order parameter, $\overline{P_{2}}$, for the spin probe 2 in the nematic and smectic $A$ phases of the mesogen 1 on the shifted temperature $\left(T_{\mathrm{N} I}-T\right)$.

nematic transition there is a jump in the order parameter of approximately 0.1 , although a certain pre-transitional rounding at the transition is observed. The large discontinuity in $\overline{P_{2}}$ is consistent with the high entropy of transition, $\Delta S_{\mathrm{S}_{\mathrm{A}} \mathrm{N}} / R$, which we have measured to be 0.66 and which should be compared with the smaller value of 0.17 found for the nematic-isotropic transition; in contrast, the entropy of melting, $\Delta S_{\mathrm{CS}_{\mathrm{A}}} / R$, is measured to be 6.80 . These observations are in accord with the predictions of molecular field theories for smectogens, when the nematic range is relatively short. ${ }^{15}$ The results for the discontinuity in the orientational order parameter and the entropy, together with the short nematic range, suggest that the transitional order makes a large contribution to the single particle potential. ${ }^{15}$ Such a contribution is in accord with other studies of smectogens. ${ }^{16}$ Once within the smectic A phase, the order parameter, $\overline{P_{2}}$, changes less rapidly with temperature; this behaviour is also in agreement both with experimental studies, ${ }^{7,14}$ and with the predictions of theory. ${ }^{15}$

Angular variations of the hyperfine spacing. Rotation of the sample in the nematic phase produces an instantaneous change in the angle made by the director with the magnetic field. However, the magnetic torque on the director rapidly restores its alignment parallel to the field, provided the anisotropy in the magnetic susceptibility is 


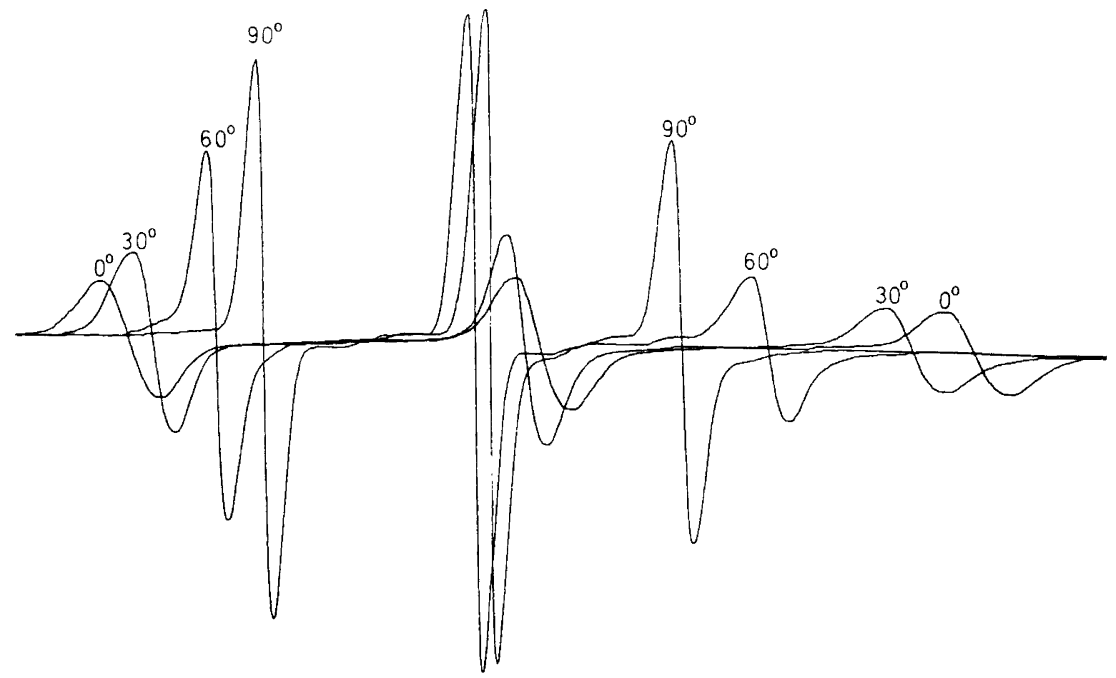

Fig. 3. The angular variation of the ESR spectrum of the spin probe 2 dissolved in the smectic A phase of the mesogen 1 at $104.6^{\circ} \mathrm{C}$. The numbers labelling the low- and high-field lines of the spectra are the rotation angle, $\gamma$.

positive. This is not the case for the smectic A phase used in our experiments, for the field strength $(0.3 \mathrm{~T})$ is insufficient to change the transitional distribution of molecules within the phase. In consequence, rotation of the sample results in a permanent change to the orientation of the director with respect to the magnetic field. The angular variation of the ESR spectrum of 2 dissolved in the smectic A phase of 1 resulting from such changes is illustrated in Fig. 3. As we can see, both the line positions and their widths vary dramatically with the director alignment; we shall consider the angular variation of the linewidths in the following section; here, we are concerned with the information which can be obtained from the line positions. According to theory, ${ }^{17}$ the hyperfine spacing, $\tilde{a}(\gamma)$, when the director makes an angle $\gamma$ with the magnetic field is

$\tilde{a}(\gamma)=\left(\tilde{A}_{\|}^{2} \cos ^{2} \gamma+\tilde{A}_{\perp}^{2} \sin ^{2} \gamma\right)^{1 / 2}$,

provided the small anisotropy in the partially averaged $g$ tensor is ignored. As we have seen, $\tilde{A}_{\|}$ is the component of the partially averaged hyperfine tensor parallel to the director and $\tilde{A}_{\perp}$ is the component perpendicular to the director. For a smectic A phase the layers are originally formed orthogonal to the field, and so the director is parallel to this with $\gamma=0^{\circ}$. Rotation of the sample about an axis perpendicular to the field therefore rotates the director through the same angle. Accordingly, the extrema in the splitting $\tilde{a}(\gamma)$ occur when $\gamma$ is equal to $0^{\circ}$ or $90^{\circ}$. This is not the situation for a tilted phase, such as a smectic $\mathrm{C}$, for which the extrema are shifted by an amount equal to the tilt angle, at least for a monodomain sample..$^{6,17}$

The angular dependence of the nitrogen hyperfine spacing is shown in Fig. 4 for the range $0 \leq \gamma \leq 180^{\circ}$; as we can see, the minimum occurs when $\gamma$ is $90^{\circ}$, with associated maxima at $0^{\circ}$ and $180^{\circ}$. The angular variation of $\tilde{a}(\gamma)$ predicted by eqn. (4) with $\tilde{A}_{\|}=2.18 \mathrm{mT}$ and $\tilde{A}_{\perp}=1.06 \mathrm{mT}$ is shown as the dotted line in Fig. 4 , and is in complete agreement with experiment. This observation is consistent with the previous identification of the smectic phase as an A, based on optical and miscibility studies. ${ }^{5}$ Knowledge of the components of the partially averaged nitrogen hyperfine tensor, $\tilde{A}$, allows us to check our assumption that the scalar coupling in the liquid crystal phase can be identified with the hyperfine interaction in the isotropic phase. Thus, the scalar coupling is related to $\tilde{A}$ by

$a=\left(\tilde{A}_{\|}+2 \tilde{A}_{\perp}\right) / 3$

and substitution of the components determined from our measurements in the smectic A phase 


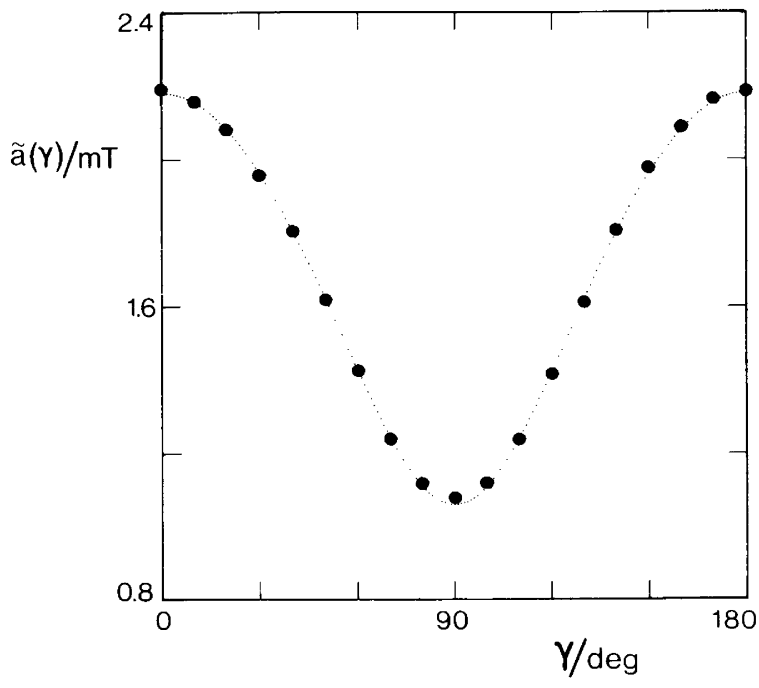

A SPIN-LABELLED LIQUID CRYSTAL

Fig. 4. The dependence of the nitrogen hyperfine splitting, $\tilde{a}(\gamma)$, for the spin probe 2 in the smectic A phase of 1 at $104.6^{\circ} \mathrm{C}$ on the angle $\gamma$ between the director and the field. The dotted line shows the angular dependence predicted by eqn. (4) with $\tilde{A}_{\|}=2.18 \mathrm{mT}$ and $\tilde{A}_{\perp}=1.06 \mathrm{mT}$. gives $a$ as $1.43 \mathrm{mT}$, in perfect accord with the isotropic value of $1.431 \mathrm{mT}$ determined for the spin probe.

The linewidths - theory. We begin our discussion of the linewidths and their angular variation with a brief description of the theory which is needed to interpret our results. For a spin probe in which the unpaired electron interacts with a single nucleus of spin $I$, the spectral lineshape is lorentzian. The width, $T_{2}^{-1}$, of this lorentzian is related to the nuclear spin quantum number, $m$, associated with each hyperfine line by

$T_{2}^{-1}=\mathrm{A}+\mathrm{B} m+\mathrm{Cm}^{2}$,

provided the dominant spin relaxation process is the rotational modulation of the laboratory components of the $g$ and hyperfine tensors. ${ }^{18}$ This expression also applies when the probe moves within an anisotropic environment, such as a liquid crystal, but now the linewidth coefficients A, $B$ and $C$ depend on the angle $\gamma$ between the director and the magnetic field. ${ }^{2}$ This angular dependence is predicted to be

$$
\mathrm{A}(\gamma)=\mathrm{A}_{0}+\mathrm{A}_{2} P_{2}(\cos \gamma)+\mathrm{A}_{4} P_{4}(\cos \gamma)
$$

with analogous expressions for B and C. Strictly speaking, these expressions are only valid when the electron and nuclear spins are quantised along the applied magnetic field. ${ }^{19}$ However, we shall not be concerned with this additional complication to our analysis which, as we shall see, is already somewhat involved.

The angle-dependent linewidth coefficients are governed by the orientational order as well as the rotational dynamics. The simplest expressions are obtained when the strong collision model is used to describe the molecular dynamics ${ }^{2,20}$ although similar forms may be obtained with the diffusional model developed by Nordio and his colleagues. ${ }^{21}$ With the strong collision model the angle-dependent linewidth coefficients are given by eqns. (8)-(16). In these somewhat forbidding expressions the $g$ and hyperfine tensors are written in their irreducible, spherical tensor forms in a common molecular frame with the $z$ axis parallel to the effective molecular symmetry axis. The orientational order parameters for this axis are $\overline{P_{2}}$ and $\overline{P_{4}}$; we see, therefore, that the linewidths contain new information concerning the ordering of the spin probe, in the form of the fourth rank order parameter. The dynamic information is provided by the three rotational correlation times, $\tau_{p}$, which may be defined in terms of the time correlation functions of the Wigner rotation matrices. ${ }^{2,20,22}$ 
LUCKHURST ET AL.

$$
\begin{aligned}
& \mathrm{A}_{0}=\frac{2 \omega_{0}^{2}}{15 g^{2}}\left\{\mathrm{~F}_{\mathrm{g}}^{(2,0)} \mathrm{F}_{\mathrm{g}}^{(2,0) *}\left(1-\overline{P_{2}^{2}}\right) \tau_{0}+2 \mathrm{~F}_{\mathrm{g}}^{(2,1)} \mathrm{F}_{\mathrm{g}}^{(2,1) *} \tau_{1}+2 \mathrm{~F}_{\mathrm{g}}^{(2,2)} \mathrm{F}_{\mathrm{g}}^{(2,2) *} \tau_{2}\right\} \\
& +\frac{\mathrm{I}(\mathrm{I}+1)}{20}\left\{\mathbf{F}_{\mathrm{A}}^{(2,0)} \mathbf{F}_{\mathrm{A}}^{(2,0) *}\left(1-\overline{P_{2}^{2}}\right) \tau_{0}+2 \mathrm{~F}_{\mathrm{A}}^{(2,1)} \mathrm{F}_{\mathrm{A}}^{(2,1) *} \tau_{1}+2 \mathrm{~F}_{\mathrm{A}}^{(2,2)} \mathrm{F}_{\mathrm{A}}^{(2,2) *} \tau_{2}\right\} \\
& \mathrm{A}_{2}=\frac{4 \omega_{0}^{2} \overline{P_{2}}}{21 g^{2}}\left\{\mathrm{~F}_{\mathrm{g}}^{(2,0)} \mathrm{F}_{\mathrm{g}}^{(2,0) *}\left(1-\overline{P_{2}}\right) \tau_{0}+\mathrm{F}_{\mathrm{g}}^{(2,1)} \mathrm{F}_{\mathrm{g}}^{(2,1) *} \tau_{1}-2 \mathrm{~F}_{\mathrm{g}}^{(2,2)} \mathrm{F}_{\mathrm{g}}^{(2,2) *} \tau_{2}\right\} \\
& +\frac{\mathrm{I}(\mathrm{I}+1) \overline{P_{2}}}{28}\left\{\mathrm{~F}_{\mathrm{A}}^{(2,0)} \mathrm{F}_{\mathrm{A}}^{(2,0) *}\left(1-\overline{P_{2}}\right) \tau_{0}+\mathrm{F}_{\mathrm{A}}^{(2,1)} \mathrm{F}_{\mathrm{A}}^{(2,1) *} \tau_{1}-2 \mathrm{~F}_{\mathrm{A}}^{(2,2)} \mathrm{F}_{\mathrm{A}}^{(2,2) *} \tau_{2}\right\} \\
& \mathrm{A}_{4}=\frac{4 \omega_{0}^{2}}{35 g^{2}}\left\{3 \mathrm{~F}_{\mathrm{g}}^{(2,0)} \mathrm{F}_{\mathrm{g}}^{(2,0) *}\left(\overline{P_{4}}-\overline{P_{2}^{2}}\right) \tau_{0}-4 \mathrm{~F}_{\mathrm{g}}^{(2,1)} \mathrm{F}_{\mathrm{g}}^{(2,1) *} \overline{P_{4}} \tau_{1}+\mathrm{F}_{\mathrm{g}}^{(2,2)} \mathrm{F}_{\mathrm{g}}^{(2,2) *} \overline{P_{4}} \tau_{2}\right\} \\
& -\frac{\mathrm{I}(\mathrm{I}+1)}{35}\left\{3 \mathrm{~F}_{\mathrm{A}}^{(2,0)} \mathrm{F}_{\mathrm{A}}^{(2,0) *}\left(\overline{P_{4}}-\overline{P_{2}^{2}}\right) \tau_{0}-4 \mathrm{~F}_{\mathrm{A}}^{(2,1)} \mathrm{F}_{\mathrm{A}}^{(2,1) *} \overline{P_{4}} \tau_{1}+\mathrm{F}_{\mathrm{A}}^{(2,2)} \mathrm{F}_{\mathrm{A}}^{(2,2) *} \overline{P_{4}} \tau_{2}\right\} \\
& \mathrm{B}_{0}=\frac{4 \omega_{0}}{15 g}\left\{\mathrm{~F}_{\mathrm{g}}^{(2,0)} \mathrm{F}_{\mathrm{A}}^{(2,0) *}\left(1-\overline{P_{2}^{2}}\right) \tau_{0}+2 \mathrm{~F}_{\mathrm{g}}^{(2,1)} \mathrm{F}_{\mathrm{A}}^{(2,1) *} \tau_{1}+2 \mathrm{~F}_{\mathrm{g}}^{(2,2)} \mathrm{F}_{\mathrm{A}}^{(2,2) *} \tau_{2}\right\} \\
& \mathrm{B}_{2}=\frac{8 \omega_{0} \overline{P_{2}}}{21 g}\left\{\mathrm{~F}_{\mathrm{g}}^{(2,0)} \mathrm{F}_{\mathrm{A}}^{(2,0) *}\left(1-\overline{P_{2}}\right) \tau_{0}+\mathrm{F}_{\mathrm{g}}^{(2,1)} \mathrm{F}_{\mathrm{A}}^{(2,1) *} \tau_{1}-2 \mathrm{~F}_{\mathrm{g}}^{(2,2)} \mathrm{F}_{\mathrm{A}}^{(2,2) *} \tau_{2}\right\} \\
& \mathbf{B}_{4}=\frac{8 \omega_{0}}{35 g}\left\{3 \mathrm{~F}_{\mathrm{g}}^{(2,0)} \mathrm{F}_{\mathrm{A}}^{(2,0) *}\left(\overline{P_{4}}-\overline{P_{2}^{2}}\right) \tau_{0}-4 \mathrm{~F}_{\mathrm{g}}^{(2,1)} \mathrm{F}_{\mathrm{A}}^{(2,1) *} \overline{P_{4}} \tau_{1}+\mathrm{F}_{\mathrm{g}}^{(2,2)} \mathrm{F}_{\mathrm{A}}^{(2,2) *} \overline{P_{4}} \tau_{2}\right\} \\
& \mathrm{C}_{0}=\frac{1}{12}\left\{\mathrm{~F}_{\mathrm{A}}^{(2,0)} \mathrm{F}_{\mathrm{A}}^{(2,0) *}\left(1-\overline{P_{2}^{2}}\right) \tau_{0}+2 \mathrm{~F}_{\mathrm{A}}^{(2,1)} \mathrm{F}_{\mathrm{A}}^{(2,1) *} \tau_{1}+2 \mathrm{~F}_{\mathrm{A}}^{(2,2)} \mathrm{F}_{\mathrm{A}}^{(2,2) *} \tau_{2}\right\} \\
& \mathrm{C}_{2}=\frac{13 \overline{P_{2}}}{84}\left\{\mathrm{~F}_{\mathrm{A}}^{(2,0)} \mathrm{F}_{\mathrm{A}}^{(2,0) *}\left(1-\overline{P_{2}}\right) \tau_{0}+\mathrm{F}_{\mathrm{A}}^{(2,1)} \mathrm{F}_{\mathrm{A}}^{(2,1) *} \tau_{1}-2 \mathrm{~F}_{\mathrm{A}}^{(2,2)} \mathrm{F}_{\mathrm{A}}^{(2,2) *} \tau_{2}\right\} \\
& \mathrm{C}_{4}=\frac{1}{7}\left\{3 \mathrm{~F}_{\mathrm{A}}^{(2,0)} \mathrm{F}_{\mathrm{A}}^{(2,0) *}\left(\overline{P_{4}}-\overline{P_{2}^{2}}\right) \tau_{0}-4 \mathrm{~F}_{\mathrm{A}}^{(2,1)} \mathrm{F}_{\mathrm{A}}^{(2,1) *} \overline{P_{4}} \tau_{1}+\mathrm{F}_{\mathrm{A}}^{(2,2)} \mathrm{F}_{\AA}^{(2,2) *} \overline{P_{4}} \tau_{2}\right\} \\
& \mathscr{D}_{q p}^{2} \overline{(0) \mathscr{D}_{q p}^{2 *}(t)}-\overline{P_{2}^{2}} \delta_{o q} \delta_{o p}=\left\{\overline{\mathscr{X}_{q p}^{2}(0) \mathscr{D}_{q p}^{2 *}(0)}-\overline{P_{2}^{2}} \delta_{o q} \delta_{o p}\right\} \exp \left(-t / \tau_{p}\right)
\end{aligned}
$$

Here, it is the rotation matrix which is used to transform the components of a second rank tensor in the molecular frame to those in the laboratory frame. The dependence of the times on $p$ but not $q$ is a simplifying assumption of the strong collision model ${ }^{2}$ and is associated with the fact that $q$ is related to components in the laboratory frame while $p$ relates to those in the molecular frame. In addition to the unknowns $\overline{P_{4}}, \tau_{\mathrm{o}}, \tau$ and $\tau_{2}$ we also need the angles defining the orienta- tion of the molecular symmetry axis in the principal frames for the $g$ and hyperfine tensors to calculate the linewidths.

In principle there is just sufficient information available from the angle-dependent linewidth coefficients to determine these unknowns. However, we prefer to adopt a different approach to the interpretation of the linewidth coefficients. Our aim is to remove as many of the unknown quantities as possible by making reasonable as- 
sumptions and then to show that the fit to the B coefficients is consistent with that for the $\mathrm{C}$. We are unable to use the A linewidth coefficients because, as we shall see, there are contributions from other line broadening mechanisms to these. The second rank order parameter, $\bar{P}_{2}$, is available from the line positions but its fourth rank analogue is not; indeed, linewidth studies provide one of the few sources for ${\overline{P_{4}}}_{4}{ }^{2,23}$ Here, however, we shall use $\overline{P_{2}}$ to estimate $\overline{P_{4}}$; to a good approximation the singlet orientational distribution function is given by

$\mathrm{f}(\beta)=Z^{-1} \exp \left\{u_{2} P_{2}(\cos \beta)\right\}$,

where $\beta$ is the angle made by the molecular symmetry axis with the director and $Z$ normalises $f(\beta) .{ }^{24}$ In addition, this is the form of the distribution function given by the principle of maximum entropy when only the second rank order parameter is known. ${ }^{25}$ The order parameters can be calculated from the distribution function according to

$\overline{P_{\mathrm{L}}}=\int P_{\mathrm{L}}(\cos \beta) \mathrm{f}(\beta) \sin \beta \mathrm{d} \beta$

so that $\overline{P_{2}}$ can be used to determine the coefficient $u_{2}$ in eqn. (18); this may then be employed to evaluate $P_{4}$.

The rotational correlation times present more of a problem. Their number can be reduced by applying the approximate interpolation

$\tau_{1}=4 \tau_{0} \tau_{2} /\left(\tau_{0}+3 \tau_{2}\right)$

to relate $\tau_{1}$ to $\tau_{0}$ and $\tau_{2}{ }^{20}$ These last two are not known but their ratio can be estimated with reasonable accuracy from other linewidth studies. ${ }^{23,26}$ In addition, for this spin probe the form of the angular dependence of the linewidth coefficients is not particularly sensitive to the ratio $\tau_{0} / \tau_{2}$, and we shall therefore hold this constant at a value of 5 which is consistent with the shape anisotropy of the spin probe 2 as well as with similar investigations of other probes. ${ }^{23,26}$ This leaves the rotational correlation time $\tau_{0}$ associated with the motion of the molecular long axis, and the angle $\beta$ which is related to the molecular structure. In principle, $\beta$ is available from structural studies of other nitroxides; however, the linewidths are relatively sensitive to the choice of this angle. With our various assumptions the correlation time simply scales the linewidths. We shall therefore explore the influence of $\beta$ on the form of the angular variation of the linewidth coefficients $\mathrm{B}$ and $\mathrm{C}$; on the other hand, $\boldsymbol{\tau}_{0}$ will be optimised to give the best agreement with experiment for a given choice of $\beta$.

The linewidths - experiment. The spectral lineshapes observed for the spin probe are not strictly lorentzian since the unpaired electron also interacts with the proton spins as well as the spin of the nitrogen nucleus. The proton hyperfine interactions are not resolved but they do serve to broaden the nitrogen lines inhomogeneously. In consequence, the determination of the intrinsic linewidth is not straightforward and we have therefore adopted two strategies with which to extract the linewidths from the spectra. In the first of these we simply ignore the inhomogeneous broadening, an assumption which should influence the results for the A linewidth coefficient far more than for $\mathrm{B}$ and $\mathrm{C}$. The linewidths relative to that of the central line $(m=0)$ were determined from the heights, $h$, of the lines by using the relation

$T_{2}^{-1}(m) / T_{2}^{-1}(0)=\{h(0) / h(m)\}^{1 / 2}$.

The width of the central line, $T_{2}^{-1}(0)$, was measured from the separation between the extrema of the first derivative lineshape; this is equal to $(2 / \sqrt{3}) T_{2}{ }^{-1}$. The errors in the effective linewidth coefficients determined in this way are estimated to be $\pm 6 \mu \mathrm{T}$ for A and B but somewhat larger, at $\pm 12 \mu \mathrm{T}$, for $\mathrm{C}$. The angular dependence of these three linewidth coefficients determined in the range $0 \leq \gamma \leq 90^{\circ}$ is shown in Fig. 5a. The results were fitted to the angular variation predicted by eqn. (7) and the best fit to the data is shown in Fig. 5a as the solid lines. They are seen to provide a very good description of the experimental results, except perhaps for $\mathrm{C}$, where the quantisation of the nitrogen spin away from the field direction may be responsible for the slight differences between theory and experiment. ${ }^{19}$ The angular linewidth coefficients obtained by this fitting procedure are listed in Table 1.

We have calculated the theoretical dependence of the $\mathrm{B}$ and $\mathrm{C}$ linewidth coefficients on the director orientation via eqns. (7) and (11)-(16) using the approximations described in the previous section. We have adopted this approach rather than predicting only the angle-dependent linewidth 


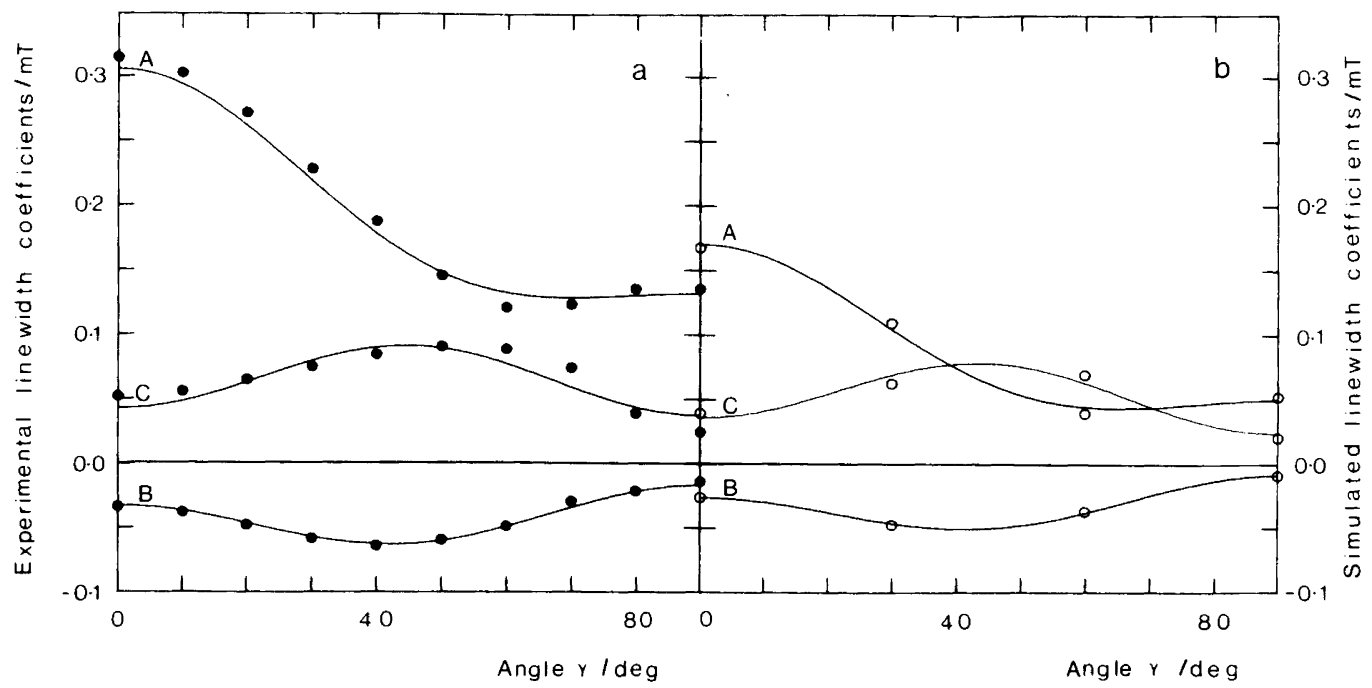

Fig. 5. The angular dependence of the linewidth coefficients $A, B$ and $C$ for the spin probe dissolved in the smectic $A$ phase of the mesogen at $104.6^{\circ} \mathrm{C}$. (a) no allowance was made for inhomogeneous broadening of the spectral lines; (b) some allowance was made via approximate simulations of the lineshapes. The solid lines show the best fit of the angular variation predicted by eqn. (7) to experiment.

coefficients since this allows us to see how the angle $\beta$ influences the theoretical predictions. The components for the anisotropic $g$ and hyperfine tensors employed in the calculations were those measured by Hubbel and McConnell $^{27}$ for an analogous nitroxide; the components are

$g_{a a}^{\prime}=-0.0035$

$g_{b h}^{\prime}=0.0033$

$g_{c c}^{\prime}=0.0002$

$$
\begin{aligned}
& A_{a a}^{\prime}=1.67 \mathrm{mT} \\
& A_{b b}^{\prime}=-0.83_{5} \mathrm{mT} \\
& A_{c c}^{\prime}=-0.83_{5} \mathrm{mT}
\end{aligned}
$$

and the scalar contributions are 2.0056 and $1.43 \mathrm{mT}$. The principal axes for the two tensors are taken to be colinear with $a$ orthogonal to the

Table 1. The angle-dependent linewidth coefficients for the spin probe in the smectic A phase extracted

\begin{tabular}{|c|c|c|c|c|c|c|}
\hline \multirow[t]{2}{*}{$\mathrm{L}$} & \multicolumn{2}{|l|}{$\mathrm{A}_{\mathrm{L}} / \mu \mathrm{T}$} & \multicolumn{2}{|l|}{$\mathrm{B}_{\mathrm{L}} / \mu \mathrm{T}$} & \multicolumn{2}{|l|}{$\mathrm{C}_{\mathrm{L}} / \mu \mathrm{T}$} \\
\hline & without & with & without & with & without & with \\
\hline 0 & 160 & 65 & -42 & -32 & 67 & 54 \\
\hline 2 & 93 & 62 & -25 & -24 & 23 & 27 \\
\hline 4 & 53 & 43 & 35 & 30 & -47 & -45 \\
\hline
\end{tabular}
from the spectra without and with allowance for the inhomogeneous broadening of the spectral lines. oxazolidine ring, $b$ parallel to the $\mathrm{N}-\mathrm{O}$ bond and $c$ perpendicular to these two. In the calculations, the angle $\beta$ between the molecular long axis of the probe and the $a$ axis was first set equal to $30^{\circ}$, the value used to extract the order parameter, $\overline{P_{2}}$, from the nitrogen hyperfine splitting. The correlation time, $\tau_{0}$, was then adjusted to obtain the best agreement with experiment. The results of these calculations are shown as the dotted lines [marked (iii)] in Fig. 6a, where they are compared with the experimental values of $B$ and $C$, indicated by the solid lines, obtained from the angular linewidth coefficients listed in Table 1 . The agreement with experiment is seen to be quite good, particularly for the $\mathrm{C}$ linewidth coefficient and especially since only a single parameter was adjusted to obtain this fit. However, the agreement is clearly not perfect and we have therefore repeated the calculations with different values $\left(24,27\right.$ and $\left.30^{\circ}\right)$ for the angle $\beta$; again, the fit to the experimental data was optimised by varying $\tau_{0}$. The results of these calculations are also shown as the dotted lines in Fig. 6a; we see that the $C$ linewidth coefficient is more sensitive to $\beta$ than $\mathrm{B}$. In addition, we find that the best fit to the C coefficient is obtained with $\beta$ equal to $24^{\circ}$, while for B a better fit is obtained with a larger value for $\beta$. The origins of this difference are not 

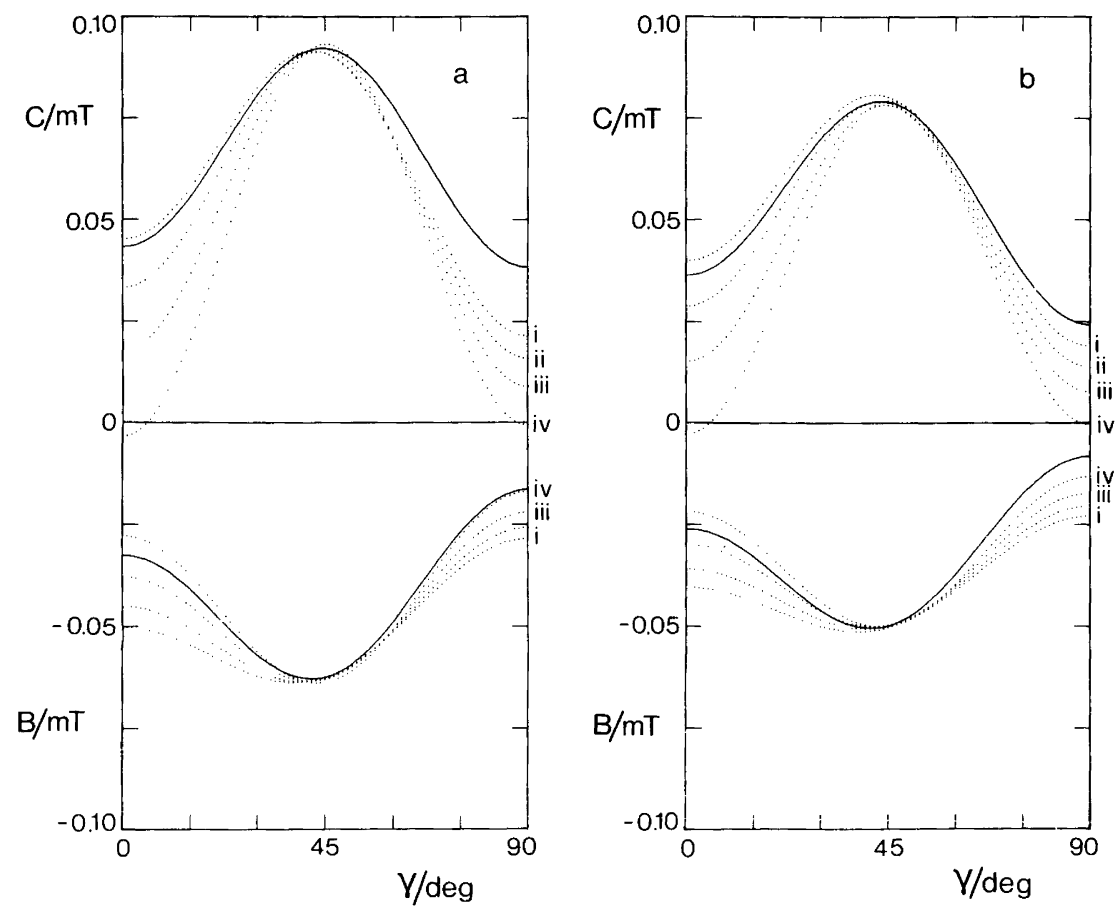

Fig. 6. A comparison of the predicted (......) and observed $(\longrightarrow$ ) angular dependence of the $\mathrm{B}$ and $\mathrm{C}$ linewidth coefficients. The calculations were made with the angle $\beta$ between the $a$ axis and the molecular long axis equal to (i) $24^{\circ}$, (ii) $27^{\circ}$, (iii) $30^{\circ}$ and (iv) $33^{\circ}$. The experimental linewidth coefficients were extracted from the ESR spectra (a) without allowing for inhomogeneous broadening and (b) with allowance made for this broadening.

clear, although it may result from our assumption concerning the quantisation of the nitrogen spin; ${ }^{19}$ however, we will not investigate this discrepancy further here. We also note that the optimum values of $\tau_{0}$ determined from the $B$ and $C$ linewidth coefficients are typically $10^{-9} \mathrm{~s} \mathrm{rad}^{-1}$ but differ by about $30 \%$. Such differences have been observed in studies of similar spin probes, ${ }^{26}$ although they are not yet understood.

Unlike the B and C linewidth coefficients, A contains a significant contribution from unresolved proton hyperfine structure. In our second method for analysing the linewidths and their angular variation we have attempted to allow for this inhomogeneous broadening in the following way: The unpaired electron in the spin probe 2 interacts with the many different protons, and allowance should be made for their particular interactions together with their angular dependence. ${ }^{27}$ This is, however, a time consuming and difficult exercise which we have simplified considerablly by making a drastic approximation. We assume that the results of the many different proton interactions may be adequately accounted for on the basis of the interaction of the unpaired electron with eleven equivalent protons. Simulation of the lineshapes for the isotropic spectrum reveals that a good fit can be obtained for the observed spectrum when the scalar coupling constant for these equivalent protons is set equal to $0.03 \mathrm{mT}$. For the smectic A phase it proved necessary to allow for the small angular dependence of the effective proton hyperfine interaction in order to obtain an equally good fit to the lineshapes. These detailed analyses of the spectral lineshapes were made at four orientations of the director with respect to the magnetic field, namely $\gamma=0,30,60$ and $90^{\circ}$. The components of the proton hyperfine tensor used in fitting the lineshape to determine the linewidths for each of the nitrogen hyperfine lines were $\tilde{A}_{\|}=0.05 \mathrm{mT}$ and $\tilde{A}_{\perp}=0.02 \mathrm{mT}$. The linewidths measured in 
Table 2. The experimental results for the angle-dependent $B$ and $C$ linewidth coefficients determined for the spin probe in the smectic A phase by allowing for the inhomogeneous broadening. The predicted values of the coefficients are given for different angles, together with the optimised rotational correlation times.

\begin{tabular}{lccccc}
\hline$\beta /{ }^{\circ}$ & 24 & 27 & 30 & 33 & Experiment \\
$\mathrm{B}_{0} / \mu \mathrm{T}$ & -39 & -37 & -35 & -33 & -32 \\
$\mathrm{~B}_{2} / \mu \mathrm{T}$ & -19 & -19 & -18 & -18 & -24 \\
$\mathrm{~B}_{4} / \mu \mathrm{T}$ & 17 & 20 & 24 & 30 & 30 \\
$10^{9} \tau_{0} / \mathrm{s} \mathrm{rad}^{-1}$ & 1.03 & 1.08 & 1.13 & 1.18 & - \\
$\mathrm{C}_{0} / \mu \mathrm{T}$ & 53 & 49 & 46 & 42 & 54 \\
$\mathrm{C}_{2} / \mu \mathrm{T}$ & 33 & 32 & 31 & 29 & 27 \\
$\mathrm{C}_{4} / \mu \mathrm{T}$ & -46 & -53 & -61 & -74 & -45 \\
$10^{9} \tau_{0} / \mathrm{s} \mathrm{rad}^{-1}$ & 1.50 & 1.53 & 1.55 & 1.58 & - \\
\hline
\end{tabular}

this way were then used to obtain the linewidth coefficients, and their angular variation is shown in Fig. 5b. As we had anticipated, allowance for the unresolved proton hyperfine structure has the greatest influence on the A linewidth coefficient, which is now considerably reduced. The changes in $\mathrm{B}$ and $\mathrm{C}$ are significantly smaller, although these coefficients also seem to be reduced in magnitude. The angular variation of $\mathrm{A}, \mathrm{B}$ and $\mathrm{C}$ was employed to determine the angle-dependent linewidth coefficients, the values of which are listed in Table 1 for comparison with the coefficients determined without allowing for inhomogeneous broadening. The orientational dependence of $\mathrm{A}$, $B$ and $C$ calculated with these coefficients is shown as the solid lines in Fig. 5b; they clearly provide a good description of our results, although this is partly to be expected because of the small number of points.

We have attempted to fit the observed angular variation of the $B$ and $C$ linewidth coefficients to theory [cf. eqns. (7) and (11)-(16)], as before, by fixing the value for the structural angle $\beta$ and then varying the rotational correlation time $\tau_{0}$. Calculations were performed with $\beta$ equal to 24 , 27,30 and $33^{\circ}$; the results of these calculations are shown in Fig. $6 \mathrm{~b}$ as the dotted lines, and the experimental values are represented by the solid lines. There is quite good agreement between theory and experiment, although, as for our previous analysis, the optimum values of $\beta$ determined by fitting the $\mathrm{B}$ and $\mathrm{C}$ linewidth coefficients are not the same. In addition, the best values for $\beta$ are $30-33^{\circ}$ from $B$ and $24-27^{\circ}$ from C; again, these are similar in magnitude to those determined previously. This difference in the optimum value of $\beta$ can also be seen by comparing the predicted angle-dependent linewidth coefficients [cf. eqns. (11)-(16)] with their experimental counterparts, and these are given in Table 2. From the results we find that the rotational correlation time $\tau_{0}$ is again about $10^{-9} \mathrm{~s} \mathrm{rad}^{-1}$, with the best value determined from B being about $40 \%$ smaller than that determined from $\mathrm{C}$.

\section{Conclusion}

The usefulness of the new spin probe which we have synthesised for studies of the static and dynamic properties of thermotropic liquid crystals has been clearly demonstrated. The nitrogen hyperfine spacing has been employed to probe the orientational order of the nematic and smectic A phases of its mesogen. However, to obtain the second rank order parameter for the long molecular axis it is necessary to know the angle between this axis and that orthogonal to the oxazolidine ring. Structural studies of other nitroxides suggest a value of $30^{\circ}$ for this angle, and we have used the angular dependence of the $\mathrm{B}$ and $\mathrm{C}$ linewidth coefficients for the spin probe in the smectic $A$ phase to confirm this result. In addition, we have determined the correlation time for rotation about the long molecular axis of the probe in the smectic A phase to be approximately $10^{-9} \mathrm{~s} \mathrm{rad}^{-1}$ from these linewidth coefficients. In future studies using such spin probes it should be possible to determine the fourth rank order parameter from the angle-dependent linewidth coefficients by taking the angle to be $30^{\circ}$.

Acknowledgements. We are grateful to the Science and Engineering Research Council for grants towards the cost of the equipment used in 
this investigation and for a studentship to $\mathrm{Mr}$. S. W. Smith. We also thank the Royal Society and the Academy of Finland for the award of a research fellowship to Dr. F. Sundholm.

\section{References}

1. See, for example, Berliner, L. J., Ed., Spin Labeling - Theory and Applications, Academic Press, New York, San Francisco and London 1976, Vol. 1; Ibid. 1979, Vol. 2; Luckhurst, G. R. In: Gray, G. W. and Winsor, P. A., Eds., Liquid Crystals and Plastic Crystals, Ellis Horwood, Chichester 1974, Vol. 2, Chap. 7.

2. Luckhurst, G. R. and Sanson, A. Mol. Phys. 24 (1972) 1297.

3. Humphries, R. L., James, P. G. and Luckhurst, G. R. Faraday Symp. 5 (1971) 107; Emsley, J. W., Hashim, R., Luckhurst, G. R., Rumbles, G. N. and Viloria, F. R. Mol. Phys. 49 (1983) 1321.

4. Carr, S. G., Khoo, S. K., Luckhurst, G. R. and Smith, H. J. Mol. Cryst. Liq. Cryst. 45 (1978) 161; Dvolaitsky, M., Paldy, F. and Taupin, C. Phys. Lett. A 45 (1973) 454.

5. Sackmann, H. and Demus, D. Z. Phys. Chem. 222 (1963) 127.

6. Luckhurst, G. R., Timimi, B. A., Pisipati, V. G. K. M. and Rao, N. V.S. Mol. Cryst. Liq. Cryst. Lett. 1 (1985) 45.

7. Luckhurst, G. R. and Setaka, M. Mol. Cryst. Liq. Cryst. 19 (1972) 179.

8. Falle, H. R. and Luckhurst, G. R. J. Magn. Reson. 3 (1970) 161.

9. See, for example, Zannoni, C. In: Luckhurst, G. R. and Gray, G. W., Eds., The Molecular Physics of Liquid Crystals, Academic Press, London, New York, San Francisco 1979, Chap. 3.

10. Emsley, J. W. and Luckhurst, G. R. Mol. Phys. 41 (1980) 19.

11. Gleason, W. B. Acta Crystallogr., Sect. B 29 (1973) 2959.
12. Luckhurst, G. R. and Sundholm, F. Mol. Phys. 21 (1971) 349.

13. Luckhurst, G. R. and Sanson, A. Mol. Cryst. Liq. Cryst. 16 (1972) 179.

14. Doane, J. W., Parker, R. S., Cvikl, B., Johnson, J. L. and Fishel, D. L. Phys. Rev. Lett. 28 (1972) 1694.

15. McMillan, W. L. Phys. Rev. A 4 (1971) 1238; Ibid. 6 (1972) 936; Kventsel, G. F., Luckhurst, G. R. and Zewdie, H. B. Mol. Phys. 56 (1985) 589.

16. Humphries, R. L. and Luckhurst, G. R. Mol. Phys. 35 (1978) 1201.

17. Luckhurst, G. R., Ptak, M. and Sanson, A. J. Chem. Soc., Faraday Trans. 2, 69 (1973) 1752.

18. See, for example, Hudson, A. and Luckhurst, G. R. Chem. Rev. 69 (1969) 191.

19. Luckhurst, G. R. and Zannoni, C. Proc. R. Soc. London A353 (1977) 87.

20. Luckhurst, G. R., Poupko, R. and Zannoni, C. Mol. Phys. 30 (1975) 499.

21. Nordio, P. L. and Segre, U. In: Luckhurst, G. R. and Gray, G. W., Eds., The Molecular Physics of Liquid Crystals, Academic Press, London, New York, San Francisco 1979, Chap. 18.

22. Beckmann, P. A., Emsley, J. W., Luckhurst, G. R. and Turner, D. L. Mol. Phys. 59 (1986) 97.

23. Luckhurst, G. R. and Poupko, R. Chem. Phys. Lett. 29 (1974) 191; Luckhurst, G. R. and Yeates, R. N. J. Chem. Soc., Faraday Trans. 2, 72 (1976) 996.

24. Luckhurst, G. R. In: Chapoy, L.L., Ed., Recent Advances in Liquid Crystalline Polymers, Elsevier Applied Science Publishers, London, New York 1985, Chap. 7.

25. Bower, D. I. J. Polym. Sci. 19 (1981) 93.

26. Luckhurst, G. R., Setaka, M. and Zannoni, C. Mol. Phys. 28 (1974) 49.

27. Luckhurst, G. R., Setaka, M. and Yeates, R. N. J. Magn. Reson. 42 (1981) 351.

Received January 16, 1987. 